\title{
COVID-19 como fuerza motriz para el desarrollo de ciudades inteligentes: el caso de Chile
}

COVID-19 as a driving force for the development of smart cities: the case of Chile

\author{
Historial del artículo \\ Recibido: \\ 18 de septiembre de 2020 \\ Revisado \\ 21 de octubre de 2020 \\ Aceptado:
}

5 de noviembre de 2020

\author{
Luis Eduardo Bastías ${ }^{\mathrm{a}}$, Pablo Pérez Leiva ${ }^{\mathrm{a}}$
}

a Diplomado Smart Cities, Facultad de Arquitectura y Urbanismo, Universidad de Chile, Chile. Correo electrónico: qz197@ uni-heidelberg.de

\section{Palabras clave}

COVID-19, ciudades inteligentes, riesgos sistémicos, smartcities.

\section{Keywords}

COVID-19, smart cities, smartcities, systemic risks.

\section{Resumen}

El objetivo del trabajo consiste en presentar una argumentación que permite concluir que la actual pandemia de COVID-19 está actuando como un catalizador de las transformaciones necesarias para que los territorios urbanos transiten hacia la conformación de ciudades inteligentes. El marco teórico empleado conjuga la noción de riesgo sistémico con la del paradigma postfordista y schumpeteriano para establecer -desde esa base teórica- que la propagación a escala mundial de la pandemia de COVID-19, durante el año 2020, ha producido un efecto acelerador de la metamorfosis del metabolismo urbano, a nivel global, la que se ha traducido en un incremento de las prácticas de transformación digital, creando así las condiciones para la evolución temprana de los territorios urbanos en ciudades inteligentes. La recolección de datos, el intercambio de información, la estimación de contagios y muertes son solo algunos elementos que pueden ser analizados y gestionados desde la perspectiva de ciudades inteligentes.

\begin{abstract}
The objective of this work is to present the rationale to conclude that the COVID-19 pandemic is acting as a catalyst of the necessary transformations for the urban territories, to transit towards the conformation of smart cities. The theoretical framework used combines the notion of systemic risk with that of the post-Fordist and Schumpeterian paradigm, to establish - from this theoretical base- that the worldwide spread of the COVID-19 pandemic, during the year 2020, has produced an accelerating effect of the metamorphosis of the urban metabolism, at a global scale, which has been translated into an increase in the practices of digital transformation, creating the conditions for the early evolution of urban territories into smart cities. Data collection, information exchange, estimation of infections and deaths, are just some elements that can be analysed and managed from that perspective of smart cities.
\end{abstract}




\section{Introducción}

La pandemia de COVID-19 es, sin duda, la situación más adversa y dramática que el mundo ha experimentado desde la Segunda Guerra Mundial. En diciembre de 2019, en la ciudad de Wuhan, capital de la provincia de Hubei, China, fueron diagnosticados los primeros casos del nuevo coronavirus de tipo 2 de síndrome respiratorio agudo severo (SARS-CoV-2) (Li et al., 2020). Esta pandemia, no solo en Chile, sino en todo el mundo, puso a prueba antiguos modelos de distribución de enfermedades y pandemias que previamente se conocían. Conocido es el caso del estudio de la epidemia de cólera en Londres del siglo XIX y el caso investigado por John Snow, el cual pudo demostrar mediante un estudio de distribución geoespacial la relación de contagios del cólera con la extracción de agua desde una bomba de uso público ubicada en Broad Street. Fue tan importante el avance de este estudio, que hasta el día de hoy Snow es considerado como "el padre de la infectología" (Cerda Lorca \& Valdivia , 2007).

En el presente estudio hemos reflexionado a partir de los aportes efectuados por el académico británico Bob Jessop (1999), y hemos encontrado allí el fundamento teórico para la irrupción de las ciudades inteligentes. Seguidamente, abordamos el tema de los riesgos sistémicos como fenómenos que se propagan más allá de las fronteras propias del sistema desde donde se inician, y demostramos que una pandemia como el COVID-19 cumple esas características.

Finalmente, observamos las consecuencias del confinamiento a gran escala, especialmente en Chile, y verificamos un aumento en la digitalización de procesos. Por otra parte, si bien, hoy en día se ha avanzado mucho gracias al desarrollo de los Sistemas de Información Geográficos (SIG), entre otros, para realizar estudios de análisis espacial en la difusión y expansión de enfermedades, y su comportamiento espacial, no fue hasta la llegada de esta pandemia en Chile que fue puesta a prueba una serie de capacidades técnicas y logísticas del mundo público, académico y privado para poder entender y predecir este comportamiento al interior de las ciudades.

\section{La crisis del Estado de Bienestar y el auge de las ciudades inteligentes}

Según el académico y escritor británico - que ha publicado extensamente sobre teoría del Estado y economía política -, Bob Jessop, "se acepta ampliamente que los regímenes de Estado de Bienestar keynesianos que surgieron durante el largo auge de la postguerra se encuentran en estado terminal" (Jessop, 1999, p. 63). Para el autor, estamos transitando desde un paradigma keynesiano y fordista a uno que él denomina postfordista y schumpeteriano. El paso de un sistema fordista a uno postfordista dice relación con la transición desde un modelo que -en última instancia- se basa en supuestos racionalistas, mecanicistas y reduccionistas de la producción, inspirados en la visión de personas como el empresario Henry Ford, el ingeniero Henri Fayol y quien es considerado como el padre de la Administración Científica, Frederick Taylor (Chiavenato, 2006). Por otra parte, los distintos objetivos del Estado de Bienestar Keynesiano (EBK) con respecto a la reproducción económica y social, "fueron promover el pleno empleo en una economía nacional relativamente cerrada" (Jessop, 1999, p. 65).

En consecuencia, el paradigma del EBK no se ajusta adecuadamente a las exigencias de la economía global, por lo que Jessop propone una nueva teoría del Estado que él denomina Estado de Trabajo Schumpeteriano (ETS), ya que así como Keynes fue citado a menudo para justificar el interés por el papel del Estado en el mantenimiento del pleno empleo, "Schumpeter está siendo redescubierto como teórico de la fuerza motivadora de la innovación en ciclos largos" (Jessop, 1999, p. 75). En este contexto, el rendimiento urbano ya no depende solo de la dotación de infraestructura de una ciudad, sino que también, y cada vez con más fuerza, de la disponibilidad y calidad de la comunicación de conocimientos y de la infraestructura social, siendo esta última forma de capital la más decisiva para la competitividad urbana (Caragliu, del Bo \& Nijkamp, 2011).

Jessop (1999) asocia esta transición, desde un EBK al ETS, a un factor clave que él denomina hollowed out y que suele traducirse como "vaciamiento" del Estado. Mediante este concepto dicho autor plantea que:

Algunas capacidades del Estado son transferidas a cuerpos pan-regionales, plurinacionales o internacionales; otras son devueltas a los niveles regionales o locales dentro del Estado nacional; y otras son asumidas por redes horizontales de poder emergentes -regionales o locales- que pasan por alto a los Estados centrales y unen regiones o localidades de diversas sociedades. Estos cambios también están asociados al desvanecimiento de las fronteras del Estado y a su creciente compromiso en estrategias de orientación social descentralizada antes que en la coordinación centralizada de carácter imperativo. (Jessop, 1999, p. 67) 
Este marco, de tipo ETS, pone de relieve la importancia de las tecnologías de la información y las comunicaciones (TIC) para mejorar el perfil competitivo de los territorios, lo que incluye la escala local, para dar así lugar al concepto de "ciudad inteligente" (smart city o smartcity), el cual se despliega como dispositivo estratégico para englobar los modernos factores de producción urbana y como estrategia para mitigar los problemas generados por el crecimiento de la población urbana y la rápida urbanización (Chourabi et al., 2012).

En términos generales, se puede decir que las características propias de una ciudad inteligente son las siguientes (Caragliu et al., 2011):

1. La utilización de la infraestructura en red para mejorar la eficiencia económica y política, y permitir el desarrollo social, cultural y urbano.

2. Un énfasis subyacente en el desarrollo urbano impulsado por empresas privadas, muchas veces multinacionales.

3. Un énfasis en el objetivo de lograr la inclusión social de diversos residentes urbanos en los servicios públicos.

4. Una insistencia en el papel crucial de las industrias de alta tecnología y creativas en el crecimiento urbano a largo plazo.

5. Atención profunda al papel del capital social y relacional en el desarrollo urbano. Una ciudad inteligente será una ciudad cuya comunidad haya aprendido a aprender, adaptarse e innovar.

6. Sostenibilidad social y ambiental como un importante componente estratégico de las ciudades inteligentes.

En efecto, un objetivo general de las ciudades inteligentes es mejorar la sostenibilidad mediante el apoyo de la tecnología (Ahvenniemi, Huovila, Pinto-Seppä \& Airaksinen, 2017).

Como resultado de lo anterior, las ciudades inteligentes emplean las tecnologías de la información y las comunicaciones para mejorar: la calidad de vida de sus ciudadanos, la economía local, el transporte, la gestión del tráfico, el medio ambiente y la interacción con el gobierno (Ismagilova, Hughes, Dwivedi \& Raman, 2019). En otras palabras, las ciudades inteligentes son aquellas en las que las Tecnologías de la Información y Comunicaciones (TIC) se fusionan con las infraestructuras tradicionales. Los desafíos que deben enfrentar las ciudades en este contexto dicen relación con la capacidad de relacionar la infraestructura con su funcionamiento operativo y la planificación urbana mediante la gestión, el control y la optimización; para así explorar la noción de la ciudad como laboratorio de innovación; con el objetivo de garantizar la equidad, la justicia y una mejor calidad de vida en la ciudad (Batty et al., 2012).

\section{Los riesgos sistémicos y sus características}

La era actual, o alta modernidad, ha sido descrita como un juggernaut, ya que todos estamos amenazados por megariesgos, los cuales han sido socialmente construidos -paradójicamente- como resultado del triunfo de la modernidad misma (Giddens, 1991). Estos riesgos son cada vez más complejos en sus causas y efectos, y operan en dimensiones mayores de espacio y tiempo (Beck, 1986). Las catástrofes ecológicas y epidemiológicas -como es el caso de una pandemia- que antiguamente eran interpretadas y asumidas como amenazas naturales, ahora tienen intrusiones, componentes y efectos de propagación que las conectan con el funcionamiento mismo de la sociedad. Una epidemia, por ejemplo, que antes podía quedar circunscrita a un territorio particular, ahora da origen a una pandemia de alcance global, que afecta a la humanidad en su conjunto. Es el caso de la peste negra, por ejemplo, la cual afectó a Europa entre 1348 y 1350 , y que dejó entre un tercio y la mitad de la población muerta, pero sin propagarse a otros territorios (Horrox, 1994). Otro ejemplo, aún más localizado, es el caso de la epidemia de cólera en Londres, ocurrida en 1854, la que afectó principalmente al sector de Soho de la capital inglesa, sin propagarse a otra áreas (Cerda Lorca \& Valdivia C., 2007).

Más aún, en el contexto de la "Sociedad del Riesgo" (Beck, 1998), los riesgos se caracterizan por la totalidad de la amenaza que representan, esto es: la posibilidad de que un sistema completo colapse como consecuencia de los mismos. Allí radica la principal diferencia entre los riesgos convencionales y los sistémicos. En una economía global, las economías locales -esto es: a nivel de Estadonación-solo pueden entenderse como módulos, o incluso nodos de una red global a la cual pertenecen y sin la cual carecen de existencia significativa (Amin \& Robins, 1990).

Si bien la noción de "riesgo sistémico" se empleó originalmente en el ámbito de la economía, el concepto ha sido utilizado no solo en dicho espacio, sino que también para referirse a riesgos ambientales y de salubridad (Briggs, 2008). Una definición de riesgo sistémico alude a la posibilidad de rupturas o quiebres en un sistema 
completo, a la diferencia de la ocurrencia de quiebres o disrupciones en sus partes individuales o componentes aislados; esto, debido a la estrecha correlación que suele haber entre la mayoría de sus elementos constituyentes (Kaufman \& Scott, 2003).

Una característica de los riesgos sistémicos es que estos se propagan más allá del dominio desde el cual surgen. Esto se traduce generalmente en la amenaza que representa para importantes servicios de la sociedad (de Bandt \& Hartmann, 2000). Desencadenante clave de este tipo de riesgo son los cambios estructurales en la sociedad actual, tales como los siguientes (Rosa, Renn \& Mccright, 2014):

1. Aumento de la población y de la densidad demográfica.

2. Aumento del consumo que amenaza al clima y al sistema de recursos planetarios.

3. Aumento de la ocupación, en zonas propensas al riesgo, para usos productivos y residenciales (Rundle, Turcotte \& Klein, 1996).

4. Aumento de la interdependencia entre los riesgos técnicos, sociales y culturales.

Todo ello hace que los pronósticos de desenlace para este tipo de riesgo sean particularmente difíciles de hacer, debido a las interacciones no lineales, altamente sensibles, propias de sistemas complejos (Klinke \& Renn, 2004; Renn, Dreyer \& Klinke, 2002; Rosa et al., 2014). Por otra parte, los riesgos sistémicos conllevan la extensión de cualquier riesgo para la salubridad y el medio ambiente, hacia un contexto más amplio de riesgos tecnológicos, financieros y económicos (Renn, 2008). Los riesgos sistémicos se encuentran en el cruce entre los eventos naturales, los desarrollos económicos sociales y tecnológicos, y las acciones políticas tanto a nivel doméstico como internacional (Rosa et al., 2014). Más aún, los riesgos sistémicos contienen tres características recurrentes que los hacen difíciles de comprender y gobernar:1) complejidad, 2) incertidumbre, y 3 ) ambigüedad (Renn et al., 2002).

Otra característica que hace que los riesgos sistémicos se diferencien de los riesgos convencionales es que sus efectos físicos negativos son algunas veces inmediatos y obvios, y en otras ocasiones sutiles y latentes. Tienen el potencial de gatillar efectos de propagación fuera del dominio en el cual la amenaza se origina (Renn, 2008). Cuando un riesgo sistémico se materializa en una calamidad, el resultado se propaga causando posiblemente consecuencias dramáticas, y efectos laterales secundarios y terciarios (Kasperson, Kasperson, Pidgeon \& Slovic, 2003). Fue el caso de la enfermedad conocida como de "las vacas locas" (encefalopatía espongiforme bovina o BSE, por sus siglas en inglés) y el desastre que causó en el Reino Unido, el cual afectó no solo a la industria agraria, sino también a la industria de los alimentos, a la economía nacional, a la salud pública y la política misma (Rosa et al., 2014). Otro ejemplo similar fue el masivo estallido de la fiebre aftosa o glosopeda que afectó a rebaños completos de ovejas en el Reino Unido en el año 2001. Expertos británicos creen que la fiebre entró con los restos de comida de un avión (Oppenheimer, 2001). Este evento tuvo un impacto catastrófico en muchas granjas, tanto familiares como industriales (Anderson, 2002), y el impacto de la enfermedad se sintió mucho más allá del ámbito de la agricultura, debido a su propagación.

La gestión de estos riesgos sistémicos involucra mucho más que simplemente mitigar sus efectos negativos directos, es necesario focalizarse en las interacciones entre los diferentes dominios de riesgos y los diferentes sistemas y territorios susceptibles de ser alcanzados. Por este motivo, los riesgos sistémicos poseen retos adicionales a los tradicionales, debido a que no son susceptibles de ser tratados de manera reduccionista, tal como ocurre con el modelo de gestión de riesgos tradicional, sino que -debido a su alta complejidad- se requiere un enfoque holístico para la identificación de los peligros, el análisis de los riesgos y para su gestión. Por otra parte, lidiar con riesgos sistémicos presenta desafíos específicos y únicos que son superiores, ya que estos varían considerablemente entre ellos y son inherentemente diferentes. Es por ello que requieren también enfoques de gobernanza fundamentalmente diferenciados para su tratamiento (van Asselt \& Renn, 2011).

En definitiva, en una era en que los riesgos están desfronterizados y existe una tendencia a que los mismos tengan una naturaleza sistémica, el despliegue a nivel global de una pandemia puede tener efectos, y va a tener efectos, en ámbitos muy distantes del origen, ya que afectará a la sociedad en su conjunto y no solo desde un punto de vista sanitario. En el caso del COVID-19, esta propagación más allá del ámbito médico ha sido consecuencia principalmente de la necesidad de establecer normas de distanciamiento físico y confinamiento a una escala mundial antes nunca vista.

\section{La pandemia de COVID-19}

El 29 de diciembre de 2019 se identificó a los primeros cuatro casos que presentaron una neumonía infecciosa, causada 
por un nuevo coronavirus, originalmente denominado 2019-nCoV; todos ellos relacionados con el mercado mayorista de pescados y mariscos de Huanan, ubicado en Wuhan, una ciudad capital de once millones de habitantes, emplazada en China meridional. Los pacientes fueron diagnosticados en los hospitales locales, mediante el mecanismo de vigilancia de la "neumonía de etiología desconocida", establecido por recomendación de la Organización Mundial de la Salud (OMS), a raíz del brote de síndrome respiratorio agudo severo (SARS) de 2003 y el síndrome respiratorio del medio oriente (MERS), con el fin de permitir la identificación oportuna de nuevos patógenos. Se descubrió un betacoronavirus, previamente desconocido, mediante el uso de una secuenciación no sesgada en muestras de pacientes con neumonía (Li et al., 2020; Zhu et al., 2020). Un caso sospechoso de COVID-19 se definió originalmente como una neumonía que cumplía los cuatro criterios siguientes:

1. Fiebre, con o sin temperatura registrada.

2. Evidencia radiográfica de neumonía.

3. Recuento de glóbulos blancos bajo o normal o recuento de linfocitos bajo.

4. Ninguna reducción de los síntomas después de un tratamiento antimicrobiano durante tres días y/o que tuviesen un vínculo epidemiológico con el Mercado Mayorista de Mariscos de Huanan o contacto con otros pacientes con síntomas similares.

Los criterios epidemiológicos para definir un caso sospechoso fueron actualizados el 18 de enero de 2020, una vez que se dispuso de nueva información sobre los casos identificados. Un paciente confirmado se definió en esa oportunidad como un caso con muestras respiratorias que dieran positivo para el SARS-CoV-2 mediante al menos uno de los tres métodos siguientes (Li et al., 2020):

\section{Aislamiento del SARS-CoV-2.}

2. Al menos dos resultados positivos por el ensayo de reacción en cadena de polimerasa de transcripción inversa en tiempo real (RT-PCR) para el SARS-CoV-2.

3. Una secuencia genética que coincida con el SARSCoV-2.

Posteriormente, se identificaron pacientes contagiados en otras ciudades chinas y luego en otros países. La rápida propagación de esta infección obedece a un patrón común que tienen todas las enfermedades infectocontagiosas, las cuales se propagan por un mecanismo cuyo modelo matemático más básico es exponencial. Dicha curva exponencial es especialmente útil durante la fase inicial de la propagación y, de hecho,: "the initial exponential growth rate of an epidemic is an important measure of the severeness of the epidemic" (Ma, 2020, p. 129). Desde que el brote de COVID-19 fue declarado una emergencia de salud pública de interés internacional (PHEIC) por la OMS (Organización Mundial de la Salud), muchos países aplicaron restricciones a los vuelos a China. Asimismo, la propia China impuso un confinamiento de la población de Wuhan, así como a toda la provincia de Hubei (Lau et al., 2020). Tras estas drásticas medidas, se observó una disminución significativa de la tasa de crecimiento y un aumento en el tiempo de duplicación de los casos. (Lau et al., 2020).

En febrero de 2020, Italia se convirtió en el epicentro de la enfermedad coronavírica (COVID-19) en Europa, con muchas exportaciones a otros países y una amplia transmisión al resto del continente. Como respuesta de salud pública, el 22 de febrero de 2020, Italia impuso un bloqueo con cierre de negocios, escuelas y lugares públicos, además de distanciamiento físico en regiones hotspot cercanas a Milán y Venecia. Aproximadamente 50.000 personas no pudieron entrar o salir de varias ciudades del Véneto y Lombardía durante catorce días sin un permiso especial. Para el 9 de marzo de 2020, se habían observado en Italia 7.375 casos confirmados en laboratorio de COVID-19 y 366 muertes, por lo que en esa fecha se amplió la cuarentena para incluir todo el norte de Italia hasta el 3 de abril. (Sjödin, Wilder-Smith, Osman, Farooq \& Rocklöv, 2020).

El objetivo de la cuarentena es contener el brote. La duración de esta depende del grado severidad de la medida: mientras más drástica sea la cuarentena, menos días se requieren para que sea efectiva, de manera que una cuarentena menos estricta dará lugar a períodos de encierro mucho más largos, que luego se volverían inviables para cualquier sociedad (Sjödin et al., 2020). No obstante, los gobiernos deben mantener un delicado equilibrio al ponderar las medidas sanitarias que consideran aplicar, teniendo en cuenta el impacto que estas podrían tener en ámbitos tan diversos como las necesidades diarias de la población, la economía, la capacidad de generación de empleos y la capacidad real de fiscalizar el cumplimiento de las medidas sanitarias adoptadas. Por lo tanto, la medida más eficaz para aplanar una curva podría ser una cuarentena total. Sin embargo, una medida tan extrema no siempre es la más eficiente, y solo debe tomarse en circunstancias extremas. En este 
contexto, el 2 de marzo de 2020, el director general de la OMS, Dr. Tedros Adhanom Ghebreyesus, señaló que "la contención del COVID-19 es factible y debe seguir siendo la máxima prioridad para todos los países. Con medidas tempranas y agresivas, los países pueden detener la transmisión y salvar vidas"1 (Adhanom Ghebreyesu, 2020). Ante esta realidad, todos los países afectados han adoptado medidas sanitarias para contener el brote, siendo la cuarentena total una de las más recurrentes.

Otro ámbito donde también la pandemia ha tenido un fuerte impacto, debido al confinamiento, es en el de la salud mental. En particular, en el caso de China se observó a consecuencia del confinamiento un aumento en los trastornos de ansiedad generalizada, síntomas depresivos y baja calidad del sueño. En particular los jóvenes, las personas que pasaban demasiado tiempo pensando en el brote y los trabajadores de la salud corrían un alto riesgo de presentar algún tipo de problema psicológicos (Huang \& Zhao, 2020). Similar situación se pudo observar en Italia, donde la prevalencia de los síntomas de la depresión y ansiedad fue del $24,7 \%$ y el $23,2 \%$, respectivamente; el $42,2 \%$ presentaba trastornos del sueño y, entre ellos, el $17,4 \%$ informaba de insomnio moderado/grave (Gualano, Lo Moro, Voglino, Bert \& Siliquini, 2020).

En este contexto, la situación de confinamiento generalizado, a nivel global, debería durar mientras no se cuente con una vacuna probada y segura que permita reducir drásticamente el nivel de contagio. No obstante, los trabajos para el desarrollo de una vacuna efectiva son extremadamente lentos y, a modo de ejemplo, uno de los estudios más prometedores, el que realiza la Universidad de Oxford en conjunto con la farmacéutica AstraZeneca, tuvo que ser interrumpido el día 8 de septiembre de 2020, debido a que uno de los participantes sufrió una enfermedad durante el proceso, por lo cual será necesario descartar que se haya producido como resultado de un efecto adverso de la vacuna aplicada (Marcano, 2020).

\section{Consecuencias del confinamiento en Chile}

La condición generalizada de confinamiento a nivel global ha significado un impacto duro a la economía mundial, debido a la disminución del volumen de las actividades comerciales afectadas. Esta situación ha afectado particularmente a Chile y, según un estudio realizado por el Hogar de Cristo, con la colaboración de Techo, así como de otras ocho instituciones, titulado "Pobreza y Pandemia: propuestas para un Chile más digno y justo", la crisis sanitaria de COVID-19 podría generar un retroceso de diez años en la lucha contra la pobreza. Según dicho estudio: "la pandemia actúa como una trampa de la pobreza, concepto que alude a la interrelación de mecanismos que impiden a un país, comunidad, hogar o persona superar su situación" (Herrera \& Gotshlich, 2020).

Por otra parte, el mal llamado "distanciamiento social" -que en realidad es distanciamiento "físico"-y los llamados al confinamiento -en general-al que obliga la emergencia sanitaria por Covid-19, ha generado importantes cambios en los hábitos de las personas, modificando radicalmente el estilo de vida. La obligación, para muchos, del teletrabajo ha llevado a grandes cantidades de chilenos a quedarse en casa y a refugiarse en Internet, no solo para trabajar, sino que también para abastecerse y entretenerse (Lara, 2020), así como para recibir educación a distancia o, simplemente, porque se ha hecho un llamado a privilegiar las plataformas digitales para hacer trámites que antes se hacían en persona (El Mostrador, 2020).

Como consecuencia de lo anterior, un importante aumento del tráfico de Internet se registró en Chile, de acuerdo con las Series Estadísticas de la Subsecretaría de Telecomunicaciones (SUBTEL), en el primer trimestre del año, las cuales dan cuenta de la importancia de la conectividad digital en el contexto generado por la pandemia de COVID-19. De acuerdo con los datos entregados por la ministra de Transportes y Telecomunicaciones, Gloria Hutt y la subsecretaría de Telecomunicaciones, Pamela Gidi, el tráfico total de Internet Fija anotó 2,8 millones de Terabytes (TB), lo que representa un aumento de $40 \%$ en comparación con los 2 millones de TB registrados en igual período del año pasado. El informe también indica que el tráfico total de Internet Móvil llegó a los 648 mil $\mathrm{TB}$, cifra $40,7 \%$ mayor con respecto de los 460 mil TB consignados a marzo de 2019 (SUBTEL, 2020).

En la misma línea, a través de un comunicado, WOM señaló que durante abril notaron un aumento del $65 \%$ en las llamadas a través de Internet (VoIP) y un crecimiento del $27 \%$ en aplicaciones de juegos en línea. En el caso de las plataformas digitales de entretenimiento, HBO se llevó la delantera con un aumento del 104\% y Netflix le siguió con un $28 \%$. Además, destacan incrementos en apps de delivery, tales como como Uber Eats (30\%) y de

\footnotetext{
${ }^{1}$ La cita textual está en inglés: "But containment of COVID-19 is feasible and must remain the top priority for all countries". La referencia puede encontrarse en la siguiente dirección web: https://www.who.int/dg/speeches/detail/who-director-general-s-opening-remarks-at-the-media-briefingon-covid-19---2-march-2020.
} 
comercio electrónico, como Amazon (64\%). Incluso las aplicaciones de citas no se quedan atrás, ya que Tinder registró un incremento del $14 \%$. Esta última abrió su servicio a nivel mundial y dejó "viajar" a miles de curiosos buscando a personas de su gusto en otras tierras. Pero al otro lado del espectro quedaron las aplicaciones de mapas y de movilización o transporte, las que cayeron un 19\%, tendencia que se espera se mantenga y profundice en el tiempo (Lara, 2020).

Por otra parte y contrario a lo que se podría suponer, durante estos meses de pandemia, la Universidad de Chile ha recibido muchas denuncias de acoso sexual. Son acusaciones en redes sociales, como publicación de fotografías íntimas e insinuaciones. Así lo revela la directora de Igualdad y Género de la Universidad de Chile, Carmen Andrade, quien anuncia aquí una segunda campaña contra la violencia de género al interior de esta casa de estudios para que las estudiantes denuncien cualquier tipo de maltrato o acoso que sufran en las redes sociales o en las plataformas para hacer las clases (Quezada, 2020).

La situación de confinamiento a nivel nacional ha hecho incluso plantear varias inquietudes acerca de la realización del plebiscito programado para octubre, tendiente a establecer o no una nueva constitución política en el país. El ministro de salud, Enrique Paris, descartó que se pueda levantar los confinamientos para efectos de facilitar esa votación, debido a que ello depende de indicadores de salud objetivos. En una entrevista a medios de prensa, la autoridad clarificó que "la Constitución asegura el derecho a voto a todos los ciudadanos de la República, eso es indesmentible. No podemos estar en contra del derecho a voto y nunca hemos estado en contra de ese derecho, solo nos corresponde velar por la salud de los ciudadanos" (Ferrer, 2020). Esta situación obligó a los distintos comandos y partidos políticos que respaldan las dos opciones -Apruebo y Rechazo- a modificar sus estrategias de campaña y volcar todos sus esfuerzos a los medios digitales y redes sociales. Por ejemplo, uno de los coordinadores de la campaña del Rechazo, el diputado Tomás Fuentes -del partido Renovación Nacional-, detalló a un medio local el despliegue que sostendrán mediante redes sociales y encuentros virtuales por Zoom, entre otras herramientas (Cerna \& Vargas, 2020).

\section{Uso de datos geolocalizados en Chile}

Como se ha planteado previamente, uno de los elementos que definen una Ciudad Inteligente o "Smart City" es el uso eficiente de datos, tanto para la planificación como para la organización de las ciudades actuales. Estos datos pueden provenir de distintas fuentes, y una de ellas son las fuentes de datos ciudadanos. Muchas veces, dependiendo de ciertas condiciones técnicas, cuando los datos provienen desde los ciudadanos, al mismo tiempo que estos son recolectados, se puede obtener la referencia espacial desde dónde fueron proporcionados. Es aquí cuando un investigador puede empezar a realizar un análisis espacial que vincule datos y situaciones que comprendan el espacio geográfico para tales efectos. Un ejemplo del uso de datos ciudadanos durante la pandemia del COVID-19 en Chile es lo realizado por la "startup" chilena Daoura, quien recolectó datos provenientes desde redes sociales como Twitter, Facebook, Instagram, noticias y blogs y logró procesar mediante técnicas de Inteligencia Artificial más de 100.000.000 de textos y vincularlos con datos espaciales de meso escala como comunas o ciudades en específico, así fue como entrenaron un proceso para categorizar datos urbanos y sociales como seguridad, salud y movilización (Daoura, 2020) (figura 1).

Si bien, hoy en día se ha avanzado mucho gracias al desarrollo de los Sistemas de Información Geográficos entre otros, para realizar estudios de análisis espacial en la difusión y expansión de enfermedades, y su comportamiento espacial, no fue hasta la llegada de esta pandemia en Chile que fue puesta a prueba una serie de capacidades técnicas y logísticas del mundo público, académico y privado para poder entender y predecir este comportamiento al interior de las ciudades.

En este sentido, es interesante observar lo realizado por distintos centros de investigación de Chile, tal como el tablero visualizador COVID-19 en Chile, desarrollado por académicos el Departamento de Geografía de la Facultad de Arquitectura y Urbanismo de la Universidad de Chile (Sarricolea et al., 2020), donde, desde el mes de abril de 2020, y semana tras semana, se ha podido revisar el conteo oficial de los datos entregados por el Ministerio de Salud del número de personas contagiadas, fallecidas y recuperadas, junto con presentar mapas coropléticos de intensidad de estos datos, para que la población pudiese entender de mejor manera el comportamiento de la pandemia y su distribución espacial (Alvarado, 2020) (figura 2).

De igual forma, se puede mencionar el esfuerzo del Ministerio de Ciencias del Gobierno de Chile, tendiente a proporcionar datos mediante tableros, los cuales pueden ser visitados en la siguiente dirección web de Internet: https://www.minciencia.gob.cl/comunidad_covid19. 


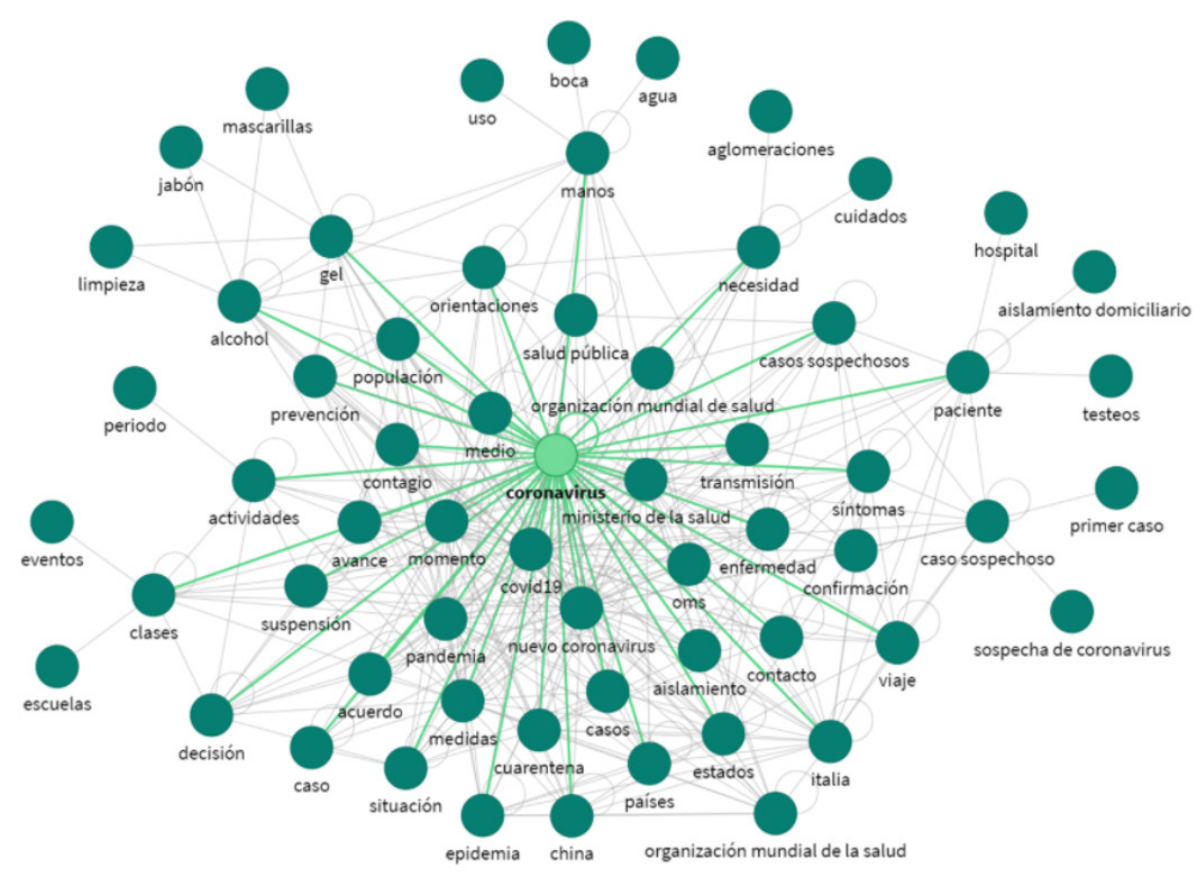

Figura 1. Grafo de las palabras encontradas en las manifestaciones sobre coronavirus y sus relaciones. Fuente: https://daoura. ai/es/blog/ignorar-o-actuar-inteligencia-artificial-en-tiempos-de-coronavirus/

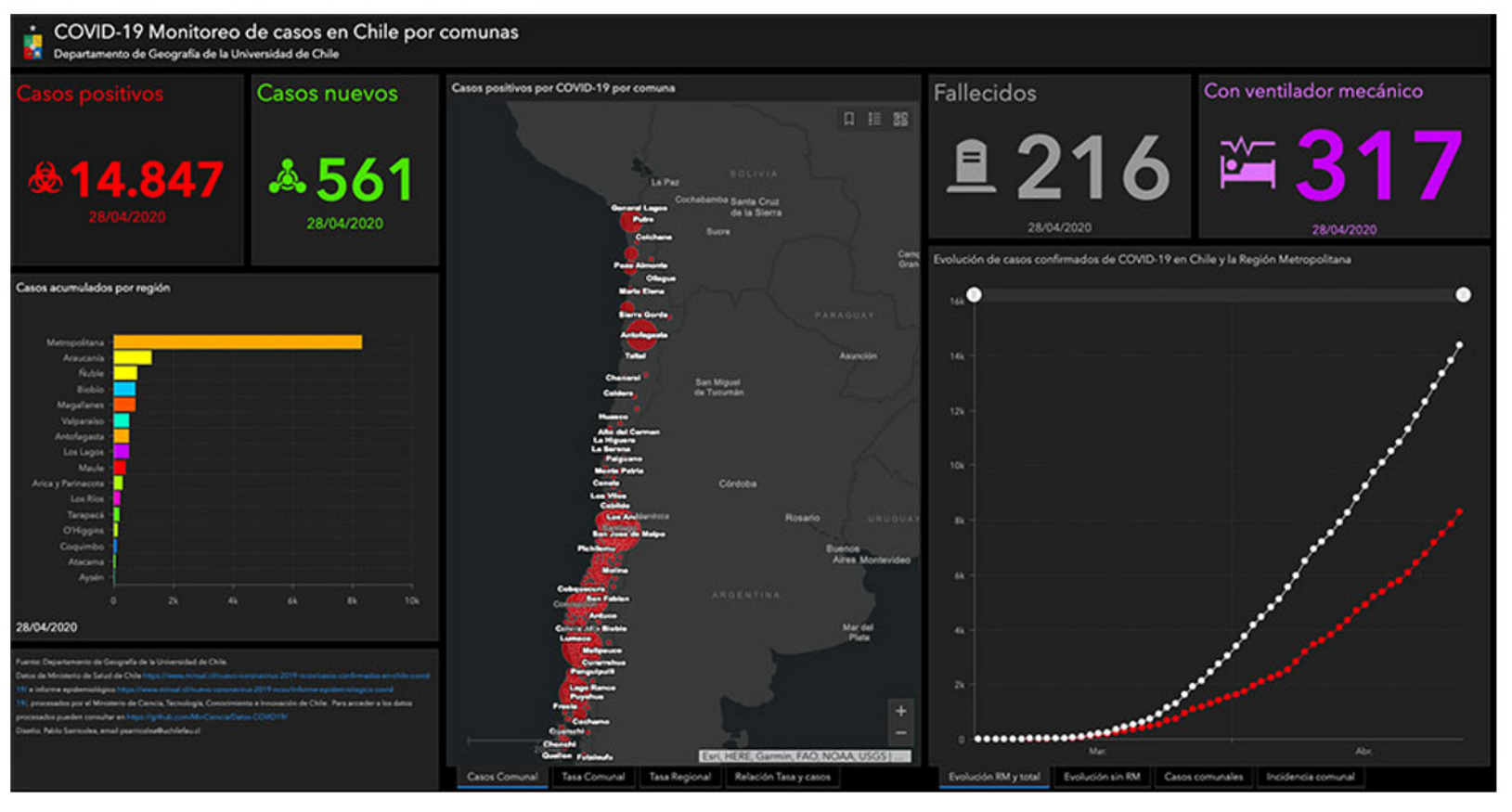

Figura 2. Tablero visualizador COVID-19 en Chile. Fuente: https://www.uchile.cl/preview/noticialmp.jsp?id=162848 


\section{Conclusiones}

La pandemia de COVID-19 es, sin duda, la situación más adversa y dramática que el mundo ha experimentado desde la Segunda Guerra Mundial. En diciembre de 2019, en la ciudad de Wuhan, capital de la provincia de Hubei, China, fueron diagnosticados los primeros casos del nuevo coronavirus de tipo 2 de síndrome respiratorio agudo severo (SARS-CoV-2) (Li et al., 2020). Esta pandemia, no solo en Chile, sino en todo el mundo, puso a prueba antiguos modelos de distribución de enfermedades y pandemias, que previamente se conocían al interior de la disciplina de la Geografía de la Salud.

En el presente artículo, hemos reflexionado a partir de los aportes efectuados por el académico y escritor británico Bob Jessop, y hemos encontrado allí un fundamento teórico para el advenimiento de las ciudades inteligentes. Seguidamente hemos abordado el tema de los riesgos sistémicos, entendidos como fenómenos que se propagan más allá de las fronteras propias del sistema desde el cual se inician, y mostramos que una pandemia como el COVID-19 cumple con todas las características propias de estos, por lo que no es extraño que a consecuencia de un brote se produzcan efectos tan distantes del origen como lo es el desarrollo de ciudades inteligentes.

Observamos también las consecuencias del confinamiento a gran escala, especialmente en Chile, deteniéndonos en el impacto que tiene en la salud mental de las personas y en los cambios de dinámicas que conlleva y que potencian el incremento en un uso de tecnologías digitales. Finalmente, verificamos que, si bien, hoy en día se ha avanzado mucho gracias al desarrollo de los Sistemas de Información Geográficos, entre otros, para realizar estudios de análisis espacial en la difusión y expansión de enfermedades y su comportamiento espacial, no fue hasta la llegada de esta pandemia en Chile que fue puesta a prueba una serie de capacidades técnicas y logísticas del mundo público, académico y privado para poder entender y predecir este comportamiento al interior de las ciudades.

En vista a los casos que hemos señalado, es posible mencionar que los datos que poseen una componente espacial están llamados a tener un lugar preponderante al momento de construir ciudades inteligentes, ya que, la conjunción entre datos personales y su relevancia espacial, como en este caso a los referidos a la pandemia del COVID-19 en Chile, son datos que pueden empujar a la construcción de ciudades más eficientes en las respuestas a este gran desafío como lo es la pandemia.
Pensando en un futuro cercano, creemos que el uso de datos vinculados a la salud de la población, y siempre y cuando se proteja la privacidad de estos, es una excelente oportunidad para que el gobierno central y las distintas unidades de la administración pública puedan disponibilizar a la comunidad científica y académica varios repositorios de "datos libres" que sean públicos y seguros, con la finalidad de que distintos investigadores puedan realizar sus propios análisis y en especial quienes velan por la espacialidad de los fenómenos humanos, sobre todo geógrafos, sociólogos, urbanistas y arquitectos, quienes dada su formación holística en la observación de fenómenos sociales, están llamados a crear y a pensar en las próximas ciudades inteligentes del mundo.

\section{Referencias}

Adhanom Ghebreyesu, T. (2020). WHO DirectorGeneral's opening remarks at the media briefing on COVID-19 - 2 March 2020. Recuperado de https:// www.who.int/dg/speeches/detail/who-directorgeneral-s-opening-remarks-at-the-media-briefingon-covid-19---2-march-2020

Ahvenniemi, H., Huovila, A., Pinto-Seppä, I. \& Airaksinen, M. (2017). What are the differences between sustainable and smart cities? Cities, 60, 234245. https://doi.org/10.1016/j.cities.2016.09.009

Alvarado, R. (2020). Equipo U. de Chile creó mapa virtual para monitorear el avance del COVID-19. Recuperado de https://www.uchile.cl/preview/ noticiaImp.jsp?id=162848

Amin, A. \& Robins, K. (1990). The re-emergence of regional economies? The mythical geography of flexible accumulation. Environment \& Planning D: Society \& Space,8(1). https://doi.org/10.1068/ d080007

Anderson, I. (2002). Foot and Mouth Disease 2001: Lessons to be Learned. London: Stationery Office.

Batty, M., Axhausen, K. W., Giannotti, F., Pozdnoukhov, A., Bazzani, A., Wachowicz, M., ... Portugali, Y. (2012). Smart cities of the future. European Physical Journal: Special Topics, 214(1), 481-518. https://doi. org/10.1140/epjst/e2012-01703-3 
Beck, U. (1986). Die Risikogesellschaft: Auf dem Weg in eine andere Moderne. Frankfurt am Main, Germany: Suhrkamp.

Beck, U. (1998). La Sociedad del Riesgo. Barcelona, Buenos Aires, México: Paidós.

Briggs, D. J. (2008). A framework for integrated environmental health impact assessment of systemic risks. Environmental Health: A Global Access Science Source, 61. https://doi.org/10.1186/1476-069X-7-61

Caragliu, A., del Bo, C. \& Nijkamp, P. (2011). Smart cities in Europe. Journal of Urban Technology, 18(2), 65-82. https://doi.org/10.1080/10630732.2011.601117

Cerda Lorca, J. \& Valdivia C., G. (2007). John Snow, la epidemia de cólera y el nacimiento de la epidemiología moderna. Revista Chilena de Infectologia, 24(4). https://doi.org/10.4067/s0716-10182007000400014

Cerna, T. \& Vargas, F. (27 de agosto de 2020). Cibercrimen advierte posibles engaños en torno al Plebiscito por auge de uso de redes sociales en campaña. EMOL. Recuperado de https://www. emol.com/noticias/Nacional/2020/08/27/996164/ Cibercrimen-enganos-Plebiscito.html

Chiavenato, I. (2006). Introducción a la Teoría General de la Administración. New York: Mc Graw Hill Interamericana.

Chourabi, H., Nam, T., Walker, S., Gil-Garcia, J. R., Mellouli, S., Nahon, K., ... Scholl, H. J. (2012). Understanding smart cities: An integrative framework. Proceedings of the Annual Hawaii International Conference on System Sciences, 2289-2297. https:// doi.org/10.1109/HICSS.2012.615

Daoura. (2020). Ignorar o actuar: inteligencia artificial en tiempos de coronavirus . Recuperado de https:// daoura.ai/es/blog/ignorar-o-actuar-inteligenciaartificial-en-tiempos-de-coronavirus/

de Bandt, O. \& Hartmann, P. (2000). Systemic Risk: A Survey. CEPR Discussion Papers.

El Mostrador. (23 de mayo de 2020). Los cambios en el uso de internet tras la pandemia. El Mostrador. Recuperado de https://www.elmostrador.cl/agendapais/2020/05/23/los-cambios-en-el-uso-de-internettras-la-pandemia/
Ferrer, C. (28 de agosto de 2020). Incertidumbre por votación de contagiados en el Plebiscito: Cómo se distribuyen los casos activos a lo largo del país. EMOL. Recuperado de https://www.emol.com/ noticias/Nacional/2020/08/28/996358/Plebiscitocomunas-cuarentena-casos-activos.html

Giddens, A. (1991). The Consequences of Modernity. Santford, CA: Stanford University Press.

Gualano, M. R., Lo Moro, G., Voglino, G., Bert, F. \& Siliquini, R. (2020). Effects of COVID-19 lockdown on mental health and sleep disturbances in Italy. International Journal of Environmental Research and Public Health, 17(13), 1-13. https://doi.org/10.3390/ ijerph17134779

Herrera, J. \& Gotshlich, D. (10 de septiembre de 2020). Informe del Hogar de Cristo advierte que crisis sanitaria podría generar retroceso de 10 años en lucha global contra la pobreza. El Mercurio, p. C1.

Horrox, R. (1994). The black death. Manchester: Manchester University Press.

Huang, Y. \& Zhao, N. (2020). Generalized anxiety disorder, depressive symptoms and sleep quality during COVID-19 outbreak in China: a web-based crosssectional survey. Psychiatry Research, 288(April). https://doi.org/10.1016/j.psychres.2020.112954

Ismagilova, E., Hughes, L., Dwivedi, Y. K. \& Raman, K. R. (2019). Smart cities: Advances in researchAn information systems perspective. International Journal of Information Management, 47(January), 88100. https://doi.org/10.1016/j.ijinfomgt.2019.01.004

Jessop, B. (1999). Crisis del Estado de Bienestar: Hacia una nueva teoría del Estado y sus consecuencias sociales. Santa Fe de Bogotá, Colombia: Siglo del Hombre Editores.

Kasperson, J. X., Kasperson, R. E., Pidgeon, N. \& Slovic, P. (2003). The social amplification of risk: Assessing fifteen years of research and theory. En N. Pidgeon, R. Kasperson, \& P. Slovic (Eds.), The Social Amplification of Risk (pp. 13-46). Cambridge: Cambridge University Press. https://doi.org/10.1017/ CBO9780511550461.002

Kaufman, G. G. \& Scott, K. E. (2003). What is systemic risk, and do bank regulators retard or contribute to 
it? Independent Review. Recuperado de https://www. independent.org/pdf/tir/tir_07_3_scott.pdf

Klinke, A. \& Renn, O. (2004). Systemic risks: a new challenge for risk management. EMBO Reports, 5 Special(June), 41-46. https://doi.org/10.1038/ sj.embor.7400227

Lara, E. (2020). Así ha mutado el uso de internet en Chile durante pandemia: más delivery y romance, menos transporte. Biobio Chile. Recuperado de https://www.biobiochile.cl/noticias/ciencia-ytecnologia/pc-e-internet/2020/05/18/asi-ha-mutadouso-internet-chile-pandemia-mas-delivery-romancemenos-transporte.shtml

Lau, H., Khosrawipour, V., Kocbach, P., Mikolajczyk, A., Schubert, J., Bania, J. \& Khosrawipour, T. (2020). The positive impact of lockdown in Wuhan on containing the COVID-19 outbreak in China. Journal of Travel Medicine, 27(3). https://doi.org/10.1093/ $\mathrm{jtm} / \mathrm{taaa} 037$

Li, Q., Guan, X., Wu, P., Wang, X., Zhou, L., Tong, Y., ... Feng, Z. (2020). Early transmission dynamics in Wuhan, China, of novel coronavirus-infected pneumonia. New England Journal of Medicine, 382(13), 1199-1207. https://doi.org/10.1056/ NEJMoa2001316

Ma, J. (2020). Estimating epidemic exponential growth rate and basic reproduction number. Infectious Disease Modelling, 5, 129-141. https://doi.org/10.1016/j. idm.2019.12.009

Marcano, J. (10 de septiembre de 2020). Las claves para entender la pausa de la vacuna de Oxford. El Mercurio, p. A10.

Oppenheimer, W. (3 de marzo de 2001). Expertos británicos creen que la fiebre aftosa entró con los restos de comida de un avión. El País. Recuperado de https://elpais.com/diario/2001/03/03/ sociedad/983574001_850215.html

Quezada, J. A. (26 de agosto de 2020). Carmen Andrade, directora de Igualdad y Género de la U. de Chile: "En pandemia se han producido nuevas formas de acoso sexual". La Tercera online. Recuperado de https://www.latercera.com/ nacional/noticia/carmen-andrade-directora-de- igualdad-y-genero-de-la-u-de-chile-en-pandemiase-han-producido-nuevas-formas-de-acoso-sexual/ PIB4HOAT2REIJKIHNYKAO36OIE/

Renn, O. (2008). Risk Governance: Coping with Uncertainty in a Complex World (Earthscan Risk in Society) Series (Vol. 53). Routledge. https://doi. org/10.1017/CBO9781107415324.004

Renn, O., Dreyer, M. \& Klinke, A. (2002). Systemic Risks: A New Challenge for Risks Management. Paris: OECD.

Rosa, E. A., Renn, O. \& Mccright, A. M. (2014). The Emergence of Systemic Risks. En The Risk Society Revisited: Social Theory and Risk Governance. Philadelphia: Temple University Press.

Rundle, J. B., Turcotte, D. L. \& Klein, W. (1996). Reduction and Predictability of Natural Disasters. Boulder, CO: Westview Press.

Sarricolea, P., Manuschevich, D., Smith, P., Romero, H., Farris,M.\&Vásquez,A.(2020).COVID-19Monitoreo de casos en Chile por comunas. Recuperado de https:// psarricolea.maps.arcgis.com/apps/opsdashboard/ index.html\#/9a2a6e34b8f54ae88fdb8e97e32b476f

Sjödin, H., Wilder-Smith, A., Osman, S., Farooq, Z. \& Rocklöv, J. (2020). Only strict quarantine measures can curb the coronavirus disease (COVID-19) outbreak in Italy, 2020. Eurosurveillance, 25(13), 1-6. https:// doi.org/10.2807/1560-7917.ES.2020.25.13.2000280

SUBTEL. (2020). Tráfico total de Internet fija y móvil crece $40 \%$ a marzo de 2020 impulsado por la pandemia de COVID-19. Recuperado de https:// www.subtel.gob.cl/trafico-total-de-internet-fija-ymovil-crece-40-a-marzo-de-2020-impulsado-por-lapandemia-de-covid-19/

van Asselt, M. \& Renn, O. (2011). Risk Governance. Risk Research, 1(4), 431-449. https://doi.org/10.108 0/13669877.2011.553730

Zhu, N., Zhang, D., Wang, W., Li, X., Yang, B., Song, J., ... Tan, W. (2020). A novel coronavirus from patients with pneumonia in China, 2019. New England Journal of Medicine, 382(8), 727-733. https://doi. org/10.1056/NEJMoa2001017 